OPEN ACCESS

Edited by:

Giuseppe Grosso,

NNEdPro Global Centre for Nutrition and Health, United Kingdom

Reviewed by:

Aida Turrini,

Consiglio Per la Ricerca in Agricoltura e l'Analisi dell'Economia Agraria

(CREA), Italy

Alessandra Lafranconi, Università degli studi di Milano

Bicocca, Italy

Alice Rosi,

Università degli Studi di Parma, Italy

${ }^{*}$ Correspondence:

Louise Seconda

I.seconda@eren.smbh.univ-paris13.fr

Specialty section

This article was submitted to

Nutrition and Environmental

Sustainability,

a section of the journal

Frontiers in Nutrition

Received: 05 March 2018

Accepted: 03 April 2018

Published: 18 April 2018

Citation:

Lacour $C$, Seconda $L$, Allès $B$ Hercberg S, Langevin B, Pointereau P, Lairon D, Baudry $J$ and Kesse-Guyot $E$ (2018) Corrigendum: Environmental Impacts of Plant-Based Diets: How Does Organic Food Consumption

Contribute to Environmental

Sustainability? Front. Nutr. 5:26.

doi: 10.3389/fnut.2018.00026

\section{Corrigendum: Environmental Impacts of Plant-Based Diets: How Does Organic Food Consumption Contribute to Environmental Sustainability?}

\author{
Camille Lacour ${ }^{1}$, Louise Seconda ${ }^{1,2 *}$, Benjamin Allès ${ }^{1}$, Serge Hercberg ${ }^{1,3}$, \\ Brigitte Langevin ${ }^{4}$, Philippe Pointereau ${ }^{4}$, Denis Lairon ${ }^{5}$, Julia Baudry ${ }^{1}$ and \\ Emmanuelle Kesse-Guyot ${ }^{1}$
}

${ }^{1}$ Equipe de Recherche en Epidémiologie Nutritionnelle (EREN), Centre d'Epidémiologie et Statistiques Sorbonne Paris Cité, INSERM (U1153), INRA (U1125), CNAM, Université Paris 13, COMUE Sorbonne Paris Cité, Bobigny, France, ${ }^{2}$ Agence de l'Environnement et de la Maîtrise de l'Energie, Angers, France, ${ }^{3}$ Département de Santé Publique, Hôpital Avicenne, Bobigny, France, ${ }^{4}$ Solagro, Toulouse, France, ${ }^{5}$ Nutrition Obésité et Risque Thrombotique (NORT), Aix Marseille Université, INRA 1260, INSERM UMR S 1062, Marseille, France

Keywords: provegetarian dietary pattern, organic food consumption, eco-friendly farming, diet-related environmental impact, sustainability

\section{A corrigendum on}

Environmental Impacts of Plant-Based Diets: How Does Organic Food Consumption Contribute to Environmental Sustainability?

by Lacour C, Seconda L, Allès B, Hercberg S, Langevin B, Pointereau P, Lairon D, Baudry J and Kesse-Guyot E (2018) Front. Nutr. 5:8. doi: 10.3389/fnut.2018.00008

There was a mistake in the values of the first column of Table $\mathbf{4}$ as published. The correct version of Table 4 appears below. The authors apologize for this mistake. This error does not change the scientific conclusions of the article in any way.

The original article has been updated.

Conflict of Interest Statement: The authors declare that the research was conducted in the absence of any commercial or financial relationships that could be construed as a potential conflict of interest.

Copyright (c) 2018 Lacour, Seconda, Allès, Hercberg, Langevin, Pointereau, Lairon, Baudry and Kesse-Guyot. This is an openaccess article distributed under the terms of the Creative Commons Attribution License (CC BY). The use, distribution or reproduction in other forums is permitted, provided the original author(s) and the copyright owner are credited and that the original publication in this journal is cited, in accordance with accepted academic practice. No use, distribution or reproduction is permitted which does not comply with these terms. 
TABLE 4 | Association between provegetarian score tertile and environmental impacts according to the level of organic food consumption, BioNutriNet study, 2014.

\begin{tabular}{|c|c|c|c|c|c|c|c|c|c|}
\hline \multirow[b]{3}{*}{ GHG emissions (CO2eq/d) } & \multicolumn{2}{|c|}{ Overall } & \multicolumn{7}{|c|}{ Level of contribution of organic food to the diet } \\
\hline & \multirow[b]{2}{*}{ Mean $^{a}$} & \multirow[b]{2}{*}{$95 \% \mathrm{CL}$} & \multicolumn{2}{|c|}{ Low (0.03) } & \multicolumn{2}{|c|}{ Medium (0.23) } & \multicolumn{2}{|c|}{ High (0.63) } & \\
\hline & & & Mean ${ }^{a}$ & $95 \% \mathrm{CL}$ & Mean $^{\mathrm{a}}$ & $95 \% \mathrm{CL}$ & Mean $^{a}$ & $95 \% \mathrm{CL}$ & \\
\hline Q1 provegetarian score & 4.56 & $(4.51-4.60)$ & 4.59 & $(4.53-4.65)$ & 4.56 & $(4.48-4.63)$ & 4.10 & (3.99-4.22) & \\
\hline Q2 provegetarian score & 4.05 & $(4.01-4.08)$ & 4.13 & $(4.08-4.18)$ & 4.05 & $(4.00-4.10)$ & 3.74 & $(3.66-3.81)$ & \\
\hline Q3 provegetarian score & 3.62 & $(3.62-3.66)$ & 3.73 & $(3.68-3.78)$ & 3.68 & $(3.63-3.74)$ & 3.34 & $(3.28-3.41)$ & \\
\hline Q4 provegetarian score & 3.23 & $(3.20-3.27)$ & 3.45 & $(3.39-3.51)$ & 3.38 & $(3.33-3.43)$ & 2.94 & $(2.89-2.99)$ & \\
\hline Q5 provegetarian score & 2.27 & $(1.33-2.29)$ & 2.93 & $(2.87-2.99)$ & 2.72 & $(2.67-2.76)$ & 2.12 & $(2.09-2.14)$ & \\
\hline$P^{\mathrm{b}}$ interaction & & & & & & & & & $<0.0001$ \\
\hline$P^{\mathrm{C}} \mathrm{Q} 1$ vs. Q2 & & & & & & & & & 0.9711 \\
\hline$P^{C}$ Q1 vs. Q3 & & & & & & & & & 0.2764 \\
\hline$P^{\mathrm{C}} \mathrm{Q} 1$ vs. Q4 & & & & & & & & & $<0.0001$ \\
\hline$P^{\mathrm{C}} \mathrm{Q} 1$ vs. Q5 & & & & & & & & & $<0.0001$ \\
\hline Cumulative energy demand (MJ/d) & Mean $^{a}$ & $95 \% \mathrm{CL}$ & Mean $^{a}$ & $95 \% \mathrm{CL}$ & Mean $^{a}$ & $95 \% \mathrm{CL}$ & Mean $^{a}$ & $95 \% \mathrm{CL}$ & \\
\hline Q1 provegetarian score & 18.55 & $(18.43-18.67)$ & 18.58 & $(18.40-18.75)$ & 18.58 & $(18.39-18.78)$ & 17.33 & $(17.05-17.63)$ & \\
\hline Q2 provegetarian score & 17.43 & $(17.33-17.53)$ & 17.62 & $(17.47-17.77)$ & 17.47 & $(17.32-17.63)$ & 16.53 & $(16.32-16.73)$ & \\
\hline Q3 provegetarian score & 16.48 & $(15.52-16.58)$ & 16.87 & $(16.70-17.04)$ & 16.62 & $(16.47-16.78)$ & 15.59 & $(15.41-15.77)$ & \\
\hline Q4 provegetarian score & 15.62 & $(15.52-15.73)$ & 16.42 & $(16.21-16.63)$ & 16.10 & $(15.93-16.27)$ & 14.62 & $(14.45-14.78)$ & \\
\hline Q5 provegetarian score & 13.29 & $(13.21-13.37)$ & 15.56 & (15.33-15.79) & 14.72 & $(14.56-14.89)$ & 12.66 & $(12.56-12.76)$ & \\
\hline$P^{\mathrm{b}}$ interaction & & & & & & & & & $<0.0001$ \\
\hline$P^{C}$ Q1 vs. Q2 & & & & & & & & & 0.9417 \\
\hline$P^{C}$ Q1 vs. Q3 & & & & & & & & & 0.1044 \\
\hline$P^{\mathrm{C}} \mathrm{Q} 1$ vs. Q4 & & & & & & & & & $<0.0001$ \\
\hline$P^{\mathrm{C}} \mathrm{Q} 1$ vs. Q5 & & & & & & & & & $<0.0001$ \\
\hline Land occupational (m2/d) & Mean $^{a}$ & $95 \% \mathrm{CL}$ & Mean $^{a}$ & $95 \% \mathrm{CL}$ & Mean $^{a}$ & $95 \% \mathrm{CL}$ & Mean $^{a}$ & $95 \% \mathrm{CL}$ & \\
\hline Q1 provegetarian score & 11.33 & $(11.14-11.41)$ & 10.94 & $(10.78-11.10)$ & 11.58 & (11.39-11.78) & 11.66 & $(11.36-11.96)$ & \\
\hline Q2 provegetarian score & 10.26 & $(10.17-10.35)$ & 9.89 & $(9.76-10.03)$ & 10.31 & $(10.17-10.45)$ & 10.64 & $(10.45-10.85)$ & \\
\hline Q3 provegetarian score & 9.34 & $(9.26-9.43)$ & 8.95 & $(8.81-9.09)$ & 9.43 & $(9.29-9.57)$ & 9.61 & (9.44-9.79) & \\
\hline Q4 provegetarian score & 8.51 & (8.42-8.60) & 8.26 & (8.10-8.43) & 8.68 & $(8.54-8.83)$ & 8.50 & (8.35-8.65) & \\
\hline Q5 provegetarian score & 6.63 & $(6.57-6.69)$ & 7.03 & $(6.87-7.19)$ & 7.09 & $(6.97-7.21)$ & 6.49 & $(6.41-6.57)$ & \\
\hline$P^{\mathrm{b}}$ interaction & & & & & & & & & $<0.0001$ \\
\hline$P^{\mathrm{C}} \mathrm{Q} 1$ vs. Q2 & & & & & & & & & 0.7782 \\
\hline$P^{\mathrm{C}} \mathrm{Q} 1$ vs. Q3 & & & & & & & & & 0.9696 \\
\hline$P^{\mathrm{C}} \mathrm{Q} 1$ vs. Q4 & & & & & & & & & 0.0111 \\
\hline$P^{C}$ Q1 vs. Q5 & & & & & & & & & $<0.0001$ \\
\hline
\end{tabular}

GHG, Greenhouse gas. Models are adjusted on sex, age, and energy intake. ${ }^{a}$ Adjusted mean were obtained with ANOVA models by level of organic food contribution in the diet. $P$-trend across the provegetarian score quintile are all $<0.0001$ and were obtained with linear contrast test by level of organic food contribution in the diet. ${ }^{b} P$ for interaction between provegetarian score quintiles and the level contribution of organic food to the diet. ${ }^{c} P$-linear trend of $Q * v . Q 1$ of provegetarian score. It reflects the linearity of the difference between the 1 st and the others quintiles of provegetarian score across the level of organic consumption. 